ISSN 1794-9831

E-ISSN 2322-7028

Vol. 13 No. 2

Jul - Dic 2016

Cúcuta, Colombia

Recibido:

25 de Enero de 2016

Aprobado:

28 de Mayo de 2016
* Regente de Farmacia, Magíster en Administración.. Docente. Universidad de los Llanos. Villavicencio, Colombia. Correo electrónico: gerardo.castano@ unillanos.edu.co

** Descriptores en Ciencias de la Salud (DeCS), en la página http:// decs.bvs.br/E/ homepagee.htm de la Biblioteca Virtual en Salud del proyecto BIREME, de la Organización Mundial de la Salud y de la Organización Panamericana de Salud.

\title{
Factores relacionados con la adquisición de medicamentos
}

\author{
Gerardo Alberto Castaño-Riobueno*
}

\section{RESUMEN}

Objetivo: identificar los factores relacionados con la adquisición de medicamentos, de los usuarios de droguerías. Materiales y Métodos: estudio descriptivo de corte transversal. La muestra fue de 138 usuarios de droguerías urbanas en una ciudad del noroccidente del departamento del Meta, mayores de 18 años, que expresaron su deseo de participar en el estudio cuando se les abordó al salir de la droguería. Se construyó un cuestionario revisado por 5 expertos. Resultados: las personas que compran medicamentos en las droguerías son, principalmente, del estrato 2; el $75 \%$ de los usuarios no recibió información sobre los productos adquiridos; el $30 \%$ leyó información del envase del medicamento, el $32.3 \%$ de los usuarios de las droguerías recomienda medicamentos para el dolor de cabeza, la automedicación en la ciudad estudiada alcanza el $78 \%$, el $21 \%$ de los usuarios se asesoran del personal de la droguería. Los grupos farmacológicos que más compran son analgésicos, antiinflamatorios, antibióticos y antigripales. Conclusiones: el gran reconocimiento del personal que labora en la droguería, como personal idóneo para recomendar el uso de medicamentos, lo debe llevar a liderar los procesos de dispensación pensando más en las personas y dejar de ver al medicamento como una mercancía.

PALABRAS CLAVE: comercialización de medicamentos, servicios comunitarios de farmacia, utilización de medicamentos**.

Para citar este artículo / To reference this article / Para citar este artigo

Castaño-Riobueno GA. Factores relacionados con la adquisición de medicamentos. Rev. cienc. cuidad. 2016; 13(2): $8-21$. 


\section{Factors related with the acquisition of medication}

Objective: identify the factors related with the acquisition of medication, from the drugstore customers. Materials and Methods: a descriptive study of cross section. The sample was of 138 customers from urban drugstores in a city on the northwest of the Meta department, older than 18, that expressed their desire to participate in the study when they were asked when leaving the store. A questionnaire was made and revised by 5 experts. Results: the people that buy medicine are, mainly from social stratum $2 ; 75 \%$ of the customers did not receive information about the products; only $30 \%$ read the information of the packaging, $32,3 \%$ of the customers from the drugstore recommend medicine used for headaches, the self-medication on the analyzed city reaches $78 \%, 21 \%$ of the customers consult the drugstore staff. The best-bought pharmacological groups are painkillers, antiinflammatory, antibiotics and flu remedies. Conclusions: the great recognition of the drugstore staff, as qualified staff, to recommend the use of medicine, should lead to the processes of dispensation thinking more about the people and stop seeing medicine as merchandise.

KEYWORDS: pharmaceutical trade, community pharmacy services, drug utilization. 
ISSN 1794-9831

E-ISSN 2322-7028 Vol. 13 No. 2 Jul - Dic 2016

Cúcuta, Colombia

\section{Fatores relacionados com a aquisição de medicamentos}

\section{RESUMO}

Objetivo: identificar os fatores relacionados com a aquisição de medicamentos, dos usuários de drogarias. Materiais e Métodos: estudo descritivo de corte transversal. A amostra foi de 138 usuários de drogarias urbanas em uma cidade do noroeste do estado de Meta, maiores de 18 anos, que expressaram seu desejo de participar no estudo quando foram entrevistados ao sair da drogaria. Construiu-se um questionário revisado por 5 peritos. Resultados: as pessoas que compraram medicamentos nas drogarias eram principalmente de classe baixa (estratificação 2); o $75 \%$ dos usuários não recebeu informação sobre os produtos adquiridos; o $30 \%$ leu informação da embalagem do medicamento, o 32,3\% dos usuários das drogarias procuram medicamentos para a dor de cabeça, a automedicação na cidade estudada alcançou o $78 \%$, o $21 \%$ dos usuários foram aconselhados pelo pessoal da drogaria. Os grupos farmacológicos que mais compraram foram analgésicos, antiinflamatórios, antibióticos e remédios para a gripe. Conclusões: o importante reconhecimento do pessoal que trabalha na drogaria, como pessoal idóneo para recomendar o uso de medicamentos, deve leva-os a liderar os processos de dispensação pensando mais nas pessoas e deixar de perceber ao medicamento como uma mercancia.

PALAVRAS-CHAVE: comercialização de medicamentos, serviços comunitários de farmácia, utilização de medicamentos. 


\section{INTRODUCCIÓN}

$\mathrm{L}$

as tendencias mundiales y la distorsión que se ha generado en el comercio de los medicamentos, al considerarlos como una mercancía (1), ha permitido que se puedan comprar (2) fácilmente aquellos que necesitan de formula médica. Lo preocupante de esto es que la compra la realizan no solamente mayores de edad enfermos, sino que se ha popularizado la compra realizada por personas sanas (3). Esta dinámica, influenciada por la automedicación, induce a la compra de medicamentos ante cualquier problema de salud, reconociendo en éstos la primera alternativa de salud, dejando a un lado los hábitos de vida saludables, pues es mucho más sencillo tomar (comprar) un medicamento (4) que asumir actividades de prevención.

Los hábitos de adquisición facilitan el consumo de los medicamentos, pudiendo generar desde una simple reacción adversa hasta problemas de salud pública, lo cual preocupa aún más, si se reconoce que el medicamento es el insumo que más se usa en el caso de una enfermedad o un simple malestar, facturando en el mercado privado de medicamentos cerca de 2.2 mil millones de dólares en Colombia, de los cuales más del $50 \%$ se vende, prescribe, dispensa o consume de manera inadecuada (5-6).

García (7) reconoce la importancia de conocer el comportamiento de la población para lograr el uso adecuado del medicamento, optimizándolo como recurso de salud. En Colombia, según el Ministerio de Protección Social, existe una automedicación del $40 \%$, el consumo de antibióticos alcanza el $3.8 \%$, el $49 \%$ compra medicamentos teniendo en cuenta experiencias previas, el $11 \%$ recibe asesoría del personal de la droguería y el $50 \%$ lee información del envase (5).

Dentro de las características socioeconómicas y demográficas más importantes de los usuarios de las droguerías en Villavicencio, se encuentra que el $46.6 \%$ de compradores de medicamentos pertenece a los estratos socioeconomicos 2 y 3 , el $21 \%$ de los que adquieren medicamentos son amas de casa, y la gran mayoría de los usuarios de las droguerías consideran su salud como buena (5-6).

Históricamente, siempre ha existido en el núcleo familiar, un experto con habilidades para sanar o recomendar tratamientos ante las enfermedades, hoy en día estos expertos pueden generan hábitos de compra que puede comprometer tanto su salud como de las personas cercanas que le consultan (8), es preciso entender que, para lograr el uso adecuado de medicamentos, es fundamental entender los diferentes hábitos y necesidades de los consumidores (9).

En Villavicencio Díaz et al. (10), afirman que la decisión de compra de los medicamentos, al interior del núcleo familiar radica en la madre y la automedicación alcanza al $73 \%$, el grupo farmacológico de mayor consumo son los analgésico y antiinflamatorios (10), estos resultados son coherentes a los obtenidos en otros estudios (1113), confirmándose que la automedicación es una práctica frecuente en el país, donde los analgésicos y antiinflamatorios no esteroideos son los medicamentos de mayor consumo; también la experiencia previa y la publicidad son criterios importantes al momento de adquirir los medicamentos.

El reconocimiento de los hábitos ${ }^{\dagger}$ y características de los usuarios de las droguerías de Villavicencio para acceder a los medicamentos contribuye al estudio de la automedicación y del autocuidado y establece, además, un punto de partida para futuras investigaciones en la región que permitan disminuir las consecuencias del uso incorrecto de los medicamentos (14-15).

El comportamiento de los usuarios de los establecimientos farmacéuticos no se ha estudiado en la región de la Orinoquia, puesto que la mayoría de estudios se ha centrado en ámbitos institucionales. Adicionalmente, en el comercio de los medicamentos convergen muchos intereses y variables, las cuales hace falta investigar (16). El establecimiento de las características sociodemográficas de la población, en este caso, permite tener una perspectiva de las personas responsables de comprar los medicamentos, para enfocar en ellas, inicialmente las acciones autocuidado responsable enfocadas en el uso adecuado de medicamentos.

\footnotetext{
$†$ Entendidos como el modo de proceder que origina tendencias instintivas.

$\$$ El concepto incluye el cuidado de uno mismo o el de un miembro de la familia y amigos.
} 
ISSN 1794-9831

E-ISSN 2322-7028

Vol. 13 No. 2

Jul - Dic 2016

Cúcuta, Colombia
En este sentido, también es vital conocer los diferentes hábitos y necesidades de los usuarios de droguerías en la ciudad de Villavicencio, implica conocer si son asesorados al momento de comprar medicamentos, si leen las etiquetas de los productos, los rangos de edad de los usuarios, que personas les han recomendado los medicamentos, y los motivos por los cuales compran el medicamento.

Así mismo, el conocer el comportamiento como una de las etapas del comercio de los medicamentos, permite identificar elementos necesarios para fortalecer programas como el de sensibilización de uso de medicamentos de la Universidad de los Llanos, que son coherentes con la estrategia nacional de uso racional de medicamentos (17-18).

\section{MATERIALES Y MÉTODOS}

Se realizó un estudio descriptivo de corte transversal. Para el tamaño de la muestra se tomaron en cuenta los 329.521 (17) habitantes del municipio de Villavicencio mayores de 18 años (19), con una prevalencia esperada del $50 \%$, un peor resultado esperado aceptable del 7 $\%$ y un intervalo de confianza del $90 \%$.

El tamaño de la muestra fue calculada con Stat Calc de Epi Info 6, obteniendo 138 usuarios de las droguerías de la zona urbana de Villavicencio, habilitadas por la Secretaria de Salud Departamental del Meta, en diciembre de 2014, que no estuvieran ubicadas en el centro de la ciudad, porque los usuarios de estas droguerías se caracterizan por comprar los medicamentos con prescripción médica y es una población flotante que no necesariamente representa el comportamiento de los habitantes de Villavicencio.

Las unidades de observación de la investigación fueron los usuarios mayores de 18 años de las droguerías urbanas que hubiesen comprado, al menos, un medicamento en una droguería y expresaran su deseo de participar en el estudio. Previo a la recolección de la información, los participantes firmaron un consentimiento informado.
Para recolectar la información de la caracterización sociodemográfica y de los hábitos de adquisición se construyó el cuestionario GESI-R01", teniendo en cuenta los objetivos planteados y la revisión de la literatura. Este cuestionario fue analizado por 5 expertos, no involucrados en el estudio, quienes revisaron la pertinencia y construcción de las preguntas e hicieron las observaciones que consideraron conveniente y que fueron tenidas en cuenta en el cuestionario definitivo. Además, se realizó una prueba piloto con 15 usuarios de droguerías que permitió hacer ajustes básicamente de forma a las preguntas y sirvió como capacitación para los encuestadores.

El instrumento se dividió en 3 apartados: caracterización, hábitos de compra y automedicación, con 5,14 y 9 preguntas respectivamente. Las alternativas de respuesta fueron marcadas de acuerdo con el valor que correspondiera al usuario en el cuestionario, en razón a la naturaleza de las preguntas que, a excepción de la correspondiente a edad, involucraba variables nominales.

Se aplicó el cuestionario GESI-R01 a los usuarios de las droguerías que compraban medicamentos, los cuales fueron abordados a la salida del establecimiento, previo permiso verbal del responsable de la droguería.

El análisis de la información se llevó a cabo mediante el software SPSS 23; se realizó análisis univariado y bivariado y se presentó la información mediante frecuencias relativas.

Esta investigación fue aprobada por el Comité de Bioética de la Universidad de los Llanos bajo acta No. 002 de abril 27 de 2015, acorde con los literales d y g del artículo 6 de la Resolución No. 008430 de 1993 del Ministerio de Salud de la Republica de Colombia (20). El estudio fue realizado con apoyo económico de la misma universidad, bajo el código FCS-5-2014. El responsable de cada droguería autorizó de forma verbal que se aborde a los usuarios para aplicar el instrumento.

ब GESI: es un grupo de investigación de la Universidad de los Llanos.

R: inicial por ser una investigación generada desde el programa Tecnología en Regencia de Farmacia.

01: consecutivo del instrumento.

Así, desde el Grupo GESI se genera un código estructurado que identifica a los instrumentos. GESI: es un grupo de investigación de la Universidad de los Llanos. 


\section{OBJETIVOS}

\section{Objetivo general}

Identificar los factores socioeconómicos y culturales relacionados con la adquisición de medicamentos, que tienen los usuarios de droguerías.

\section{Objetivos específicos}

- Caracterizar en los aspectos socioeconómico y demográfico a los usuarios de las droguerías.

- Determinar los hábitos de adquisición de medicamentos de los usuarios de las droguerías.

\section{RESULTADOS}

Los datos se recolectaron en los meses de septiembre y octubre de 2015. Se presentaron limitaciones en cuanto a la seguridad de los encuestadores, por lo cual no se pudo tomar la información de 5 droguerías.

\section{Aspectos socio económicos y demográficos}

Las personas que compran medicamentos en las droguerías pertenecen principalmente al estrato socioeconómico 2 y 3 de la ciudad investigada, los menores de 29 años realizan casi la mitad de las compras $(46.6 \%)$, los usuarios que más adquieren medicamentos tienen educación básica secundaria y educación media $(56.4 \%)$, el $21 \%$ de quienes adquieren medicamentos son amas de casa y la gran mayoría de los usuarios de las droguerías consideran su salud como buena y regular, tal como se puede a preciar en la tabla 1 .
E-ISSN 2322-7028

Vol. 13 No. 2

Jul - Dic 2016

Cúcuta, Colombia 
ISSN 1794-9831

E-ISSN 2322-7028 Vol. 13 No. 2 Jul - Dic 2016 Cúcuta, Colombia

Tabla 1. Características sociodemográficas de los usuarios de droguerías.

\begin{tabular}{|c|c|c|}
\hline \multicolumn{2}{|c|}{ Característica } & $\%$ \\
\hline \multirow{2}{*}{ Género } & Femenino & 56 \\
\hline & Masculino & 44 \\
\hline \multirow{4}{*}{ Estrato socio económico } & 1 & 15 \\
\hline & 2 & 54,3 \\
\hline & 3 & 28,3 \\
\hline & 4 & 2,4 \\
\hline \multirow{11}{*}{ Edad (años) } & Menor de 20 & 5,3 \\
\hline & $20-24$ & 21,8 \\
\hline & $25-29$ & 19,5 \\
\hline & $30-34$ & 21,1 \\
\hline & $35-39$ & 11,3 \\
\hline & $40-44$ & 8,3 \\
\hline & $45-49$ & 4,5 \\
\hline & $50-54$ & 3,8 \\
\hline & $55-59$ & 2,3 \\
\hline & $60-64$ & 1,8 \\
\hline & $70-74$ & 0,8 \\
\hline \multirow{8}{*}{ Nivel de educación } & Básica primaria sin terminar & 5,3 \\
\hline & Básica primaria & 12 \\
\hline & Básica secundaria & 26,3 \\
\hline & Educación media & 30,1 \\
\hline & Técnico & 12 \\
\hline & Tecnólogo & 8,3 \\
\hline & Profesional & 5,3 \\
\hline & Posgrado & 0,8 \\
\hline \multirow{3}{*}{ ¿Cómo considera su salud? } & Buena & 78,7 \\
\hline & Regular & 20,5 \\
\hline & Mala & 0,8 \\
\hline
\end{tabular}

Fuente: Instrumento GESI-R01, 2015.

\section{Hábitos de compra}

El $75 \%$ de los usuarios no recibió información sobre los productos que adquirió $\mathrm{y}$, aunque el 100 $\%$ adquirió medicamentos, sólo el $50 \%$ reconoce tener medicamentos almacenados en su casa; así mismo, el $30 \%$ de los usuarios manifiesta que leyó la información del envase. El $77 \%$ de los medicamentos se compra para ser administrado vía oral, mientras las soluciones inyectables constituyen apenas el $16 \%$ de las compras.

Independientemente del género, la automedicación en los usuarios encuestados alcanza el $78 \%$; el 50
$\%$ de esta automedicación ocurre en usuarios cuyo rango de edad está entre 20 y 34 años, el $62 \%$ de compradores de las droguerías también está entre los 20 y los 34 años, el $33.8 \%$ de los usuarios es influenciado por la publicidad y por experiencias propias y, adicionalmente, se evidencia que usuarios con edades comprendidas entre 20 y 39 años son aquellos que más consultan, como se evidencia en la tabla 2. 
Tabla 2. Persona que recomienda medicamento a los usuarios de las droguerías, según la edad.

\begin{tabular}{|c|c|c|c|c|c|c|}
\hline \multirow[b]{2}{*}{ Años } & \multicolumn{6}{|c|}{ ¿Quién le recomendó el medicamento? } \\
\hline & Medico & $\begin{array}{l}\text { Personal de } \\
\text { la droguería }\end{array}$ & Un familiar & $\begin{array}{l}\text { Lo leí, escuche, } \\
\text { vi en alguna } \\
\text { publicidad }\end{array}$ & $\begin{array}{l}\text { Ya sé que sirve } \\
\text { y lo había } \\
\text { probado antes }\end{array}$ & $\begin{array}{c}\text { Un } \\
\text { curandero } \\
\text { o un brujo }\end{array}$ \\
\hline$>20$ & & $2,3 \%$ & $1,5 \%$ &, $8 \%$ &, $8 \%$ & \\
\hline $20-24$ & $6,8 \%$ & $3,8 \%$ & $4,5 \%$ & $2,3 \%$ & $4,5 \%$ & \\
\hline $25-29$ & $3,0 \%$ & $3,8 \%$ & $5,3 \%$ &, $8 \%$ & $6,8 \%$ & \\
\hline $30-34$ & $3,8 \%$ & $6,0 \%$ & $3,0 \%$ & $1,5 \%$ & $6,8 \%$ & \\
\hline $35-39$ &, $8 \%$ & $2,3 \%$ & $4,5 \%$ & $1,5 \%$ & $2,3 \%$ & \\
\hline $40-44$ & $1,5 \%$ & $1,5 \%$ & $2,3 \%$ &, $8 \%$ & $2,3 \%$ & \\
\hline $45-49$ & $2,3 \%$ & & & & $2,3 \%$ & \\
\hline $50-54$ & $1,5 \%$ &, $8 \%$ &, $8 \%$ & &, $8 \%$ & \\
\hline $55-59$ & $2,3 \%$ & & & & & \\
\hline $60-64$ & &, $8 \%$ & & & &, $8 \%$ \\
\hline $70-74$ & & & & & &, $8 \%$ \\
\hline Total & $21,8 \%$ & $21,1 \%$ & $21,8 \%$ & $7,5 \%$ & $26,3 \%$ & $1,5 \%$ \\
\hline
\end{tabular}

Fuente: Instrumento GESI-R01, 2015.

El $30 \%$ de los usuarios lee la información del envase. los usuarios compra medicamentos por experiencias El $85 \%$ de las personas que acuden al médico está previas (propias o de terceros), tal como se observa satisfecho con la orientación recibida, el $34 \%$ de en la tabla 3.

Tabla 3. Personas que recomiendan medicamentos y motivos de compra de los usuarios de droguerías.

\begin{tabular}{|c|c|c|c|}
\hline \multicolumn{2}{|c|}{ Quien le recomienda el medicamento (\%) } & \multicolumn{2}{|l|}{ Motivos para comprar el medicamento (\%) } \\
\hline \multirow{3}{*}{ Ya sé que sirve y lo había probado antes } & \multirow{3}{*}{26} & Había sufrido lo mismo antes y le recomendaron ese medicamento & 18,0 \\
\hline & & Sabe que sirven por publicidad & 6,0 \\
\hline & & $\begin{array}{l}\text { Conoció a alguien que había sufrido lo mismo y le recomendaron ese } \\
\text { medicamento }\end{array}$ & 2,0 \\
\hline \multirow{2}{*}{ Médico } & \multirow{2}{*}{22} & Está satisfecho con el tratamiento que le oriento el medico & 17,0 \\
\hline & & El médico le explico para qué son los medicamentos & 5,0 \\
\hline \multirow{4}{*}{ Un familiar, vecino o un conocido } & \multirow{4}{*}{22} & $\begin{array}{l}\text { Es una persona que sufrió algo parecido a lo mío y con este medicamento } \\
\text { se mejoró }\end{array}$ & 16,0 \\
\hline & & Es una persona que sabe de medicamentos & 3,0 \\
\hline & & $\begin{array}{c}\text { Esa persona sabe que el medicamento sirve porque lo escuchó o por } \\
\text { propaganda }\end{array}$ & 2,0 \\
\hline & & No sé & 1,0 \\
\hline \multirow{2}{*}{ Personal de la droguería } & \multirow{2}{*}{21} & Me ahorro el tiempo y el dinero de la consulta & 13,0 \\
\hline & & El médico y el personal de la droguería son igual de efectivo & 8,0 \\
\hline Lo leí, escuche, ví en alguna publicidad & 7,5 & Televisión & 7,5 \\
\hline Un curandero o un brujo & 1,5 & El brujo es igual o más efectivo que los médicos & 1,5 \\
\hline
\end{tabular}

Fuente: Instrumento GESI-R01, 2015. 
ISSN 1794-9831

E-ISSN 2322-7028

Vol. 13 No. 2

Jul - Dic 2016

Cúcuta, Colombia
Se puede afirmar que los grupos farmacológicos que más compran los usuarios de las droguerías del municipio son los analgésicos, los antiinflamatorios, los antibióticos y los antigripales, que equivalen a un $67 \%$ de las compras. De los anteriores, llama la atención los antibióticos que, con un $14 \%$, integran el tercer grupo farmacológico que más se consume $\mathrm{y}$, de estos, cerca del $50 \%$ son recomendados por un familiar, vecino o amigo. Los grupos farmacológicos que menos se adquieren (porcentaje inferior al $1 \%$ ) son los anti-pruriginosos, diuréticos, anti-gotosos, cortico-esteroides, vasoconstrictores, antihelmínticos, antieméticos y broncodilatadores, tal como se aprecia en la tabla 4.

Tabla 4. Porcentaje de compra de grupo farmacológico adquirido por usuarios de droguerías.

\begin{tabular}{|l|l|}
\hline \multicolumn{1}{|c|}{ Grupo Farmacológico } & Total \\
\hline Analgésico & $29 \%$ \\
\hline Antiinflamatorio & $14 \%$ \\
\hline Antibiótico & $14 \%$ \\
\hline Antigripal & $10 \%$ \\
\hline Antihipertensivo & $6 \%$ \\
\hline Mucolítico & $4 \%$ \\
\hline Antihistamínico & $3 \%$ \\
\hline Antiácido & $3 \%$ \\
\hline Antidiarreico & $3 \%$ \\
\hline Anticonceptivo & $2 \%$ \\
\hline Naturista & $2 \%$ \\
\hline Antimicótico & $2 \%$ \\
\hline Relajante muscular & $2 \%$ \\
\hline Vitamina & $2 \%$ \\
\hline
\end{tabular}

Fuente: Instrumento GESI-R01, 2015.

El $38 \%$ de los usuarios acude a los establecimientos farmacéuticos a comprar medicamentos para dolores (indefinido $17 \%$, muscular $7 \%$ y de estómago $6 \%$, entre otros) y sólo el $5 \%$, con un p valor de 0.785 , reconoce que ha tenido alguna reacción adversa independientemente de quien le recomendó el medicamento.

El $29 \%$ de los compradores menores de 20 años adquiere anticonceptivos. Los usuarios de las droguerías con edades entre 20 y 24 años compran principalmente analgésicos y antigripales (34\% y $21 \%$ respectivamente) y los grupos etarios que más compran antibióticos son 35-39 y 45-49 años (33 \% cada uno).
Los analgésicos que más consumen son ibuprofeno y diclofenaco ( $8 \%$ cada uno), buscapina (5\%). El antibiótico que más consume es la ampicilina, con un $4 \%$, siendo el cuarto medicamento que más se compra.

Los usuarios de las droguerías principalmente recomiendan -lo hacen con otras personas, porque se creen con autoridad para hacerlo, en virtud de su supuesto conocimiento-, medicamentos para dolor de cabeza y problemas musculares. Aunque se consultó, no recomiendan medicamentos para las enfermedades de transmisión sexual ni para los riñones, como se observa en la tabla 5 . 
Tabla 5. Medicamentos que más recomiendan los usuarios de droguerías, según la enfermedad.

\begin{tabular}{|c|c|c|c|}
\hline $\begin{array}{c}\text { Recomienda } \\
\text { medicamento para }\end{array}$ & $\%$ & \multicolumn{2}{|c|}{ medicamentos que más recomienda \% } \\
\hline \multirow{3}{*}{ Dolor de cabeza } & \multirow{3}{*}{32,3} & Acetaminofén & 70 \\
\hline & & Ibuprofeno & 19 \\
\hline & & Otros & 11 \\
\hline \multirow{3}{*}{ Problemas musculares } & \multirow{3}{*}{10} & Diclofenaco & 31 \\
\hline & & Metocarbamol & 31 \\
\hline & & Otros & 38 \\
\hline \multirow{3}{*}{ Dolor de estómago } & \multirow{3}{*}{9} & N-butilbromuro de hioscina & 27,3 \\
\hline & & Ranitidina & 18,2 \\
\hline & & Otros & 54.5 \\
\hline \multirow{3}{*}{ Diarrea } & \multirow{3}{*}{8,3} & Loperamida & 81,8 \\
\hline & & Metronidazol & 9,1 \\
\hline & & Otros & 9.1 \\
\hline \multirow{2}{*}{$\begin{array}{l}\text { Hipertensión arterial } \\
\text { (HTA) }\end{array}$} & \multirow{2}{*}{4} & Enalapril & 75 \\
\hline & & Losartan & 25 \\
\hline Problemas nerviosos & 0,8 & Valeriana & 100 \\
\hline \multirow{3}{*}{ Fiebre } & \multirow{3}{*}{0,2} & Acetaminofén & 65,5 \\
\hline & & Diclofenaco & 13,8 \\
\hline & & Otros & 20.7 \\
\hline
\end{tabular}

Fuente: Instrumento GESI-R01, 2015.

Independientemente del estrato socioeconómico, el nivel educativo y de cómo consideran la salud, los compradores de medicamentos consultan al personal de la droguería para que los oriente en el uso de esos medicamentos.

\section{DISCUSIÓN}

El género femenino es el mayor usuario de las droguerías, resultado coherente con Espinoza y Jofre (21) y Salmerón et al. (22), quienes, quienes reconocen a las mujeres como las líderes naturales del autocuidado en la familia. Es importante reconocer la importancia de este cuidado, pero se debe tener en cuenta, que los medicamentos no son productos inocuos, por tal razón, se debe motivar la solicitud de asesoría al iniciar algún tratamiento farmacológico.

El gran porcentaje de compradores menores de 29 años se puede explicar por las dolencias u ocupaciones de los verdaderos consumidores, quienes envían a terceras personas a hacer las compras (empleados, hijos, conocidos). La droguería, vista como una tienda especializada, se debe enfocar más en los clientes de menor edad (23).

En Villavicencio el porcentaje de usuarios de las droguerías que tienen la costumbre de leer las etiquetas de los medicamentos es bajo, con respecto a lo reportado en el estudio realizado en 2005 en cinco ciudades colombianas, donde se reporta un $50 \%$ (5) y es significativamente menor a lo reportado en Chile y Argentina, que alcanzan porcentajes de lectura de la etiqueta superiores al $65 \%$ (24)becoming a public health problem. InAims: To determine the patterns of self-medication in customers who frequented a community pharmacy in the city of Antofagasta.। InMethods: A survey was conducted to 297 users over 18 years and with adequate mental and communicative power to answer the questions, and attending the pharmacy to buy a drug without a prescription. InResults: The study revealed that of the 297 people who practiced self-medication, $41 \%$ do it with a frequency of at least once a month. The woman 
ISSN 1794-9831

E-ISSN 2322-7028

Vol. 13 No. 2

Jul - Dic 2016

Cúcuta, Colombia practiced in $64 \%$ and the men in $36 \%$. The range of age of the studied group was between 31-50 years. The main reason to practice the self medication was to recognize symptoms (33\%. Es importante que los usuarios de los medicamentos se apropien del buen uso de estos productos, reduciendo así los errores en su uso (25).

Con respecto a la automedicación en los usuarios, es el doble con respecto al $40 \%$ reportado en el estudio de cinco ciudades colombianas (5) y al estudio de Portugal (22). Esta gran diferencia puede explicarse en la metodología del estudio, la cual no tuvo en cuenta a las droguerías del centro de la ciudad por considerar que los usuarios de éstas se caracterizan por comprar los medicamentos con prescripción médica y constituyen una población flotante que no necesariamente representa el comportamiento de los habitantes de Villavicencio.

La alta influencia de los familiares, amigos o vecinos en los usuarios de las droguerías de Villavicencio es similar a lo encontrado en el estudio de Diaz et al. (10) $(23 \%)$ y con lo publicado por Waldo (26) (33\%), mientras que es mayor de acuerdo a lo reportado en el estudio realizado en el 2005 (5) en cinco ciudades Colombianas $(9 \%)$.

Así mismo, la compra de medicamentos porque el usuario ya sabe que sirven y los había probado antes ha aumentado en un promedio similar con respecto a lo reportado por Jaramillo et al. (5) en el estudio de cinco ciudades en Colombia. Un factor que puede influenciar este evidente aumento de las orientaciones ofrecidas por el familiar, amigo o vecino es el fácil acceso a la información sobre medicamentos que, a su vez, también favorece la automedicación (9).

Con respecto a otros estudios $(17,27)$, llama la atención el alto consumo de antibióticos en la población de estudio, situación que se puede explicar por la gran influencia que tienen los familiares, amigos o vecinos al momento de adquirir medicamentos. Es necesario indagar, entonces, dónde ellos obtienen información acerca de los medicamentos, cuáles son las fuentes que consultan o si es la simple experiencia de uso de los medicamentos que se comunica de boca en boca, sin contrastar la información, pudiéndose presentar información errónea o insuficiente, elección equivocada del medicamento, interacciones o, en el caso de los antibióticos, resistencia bacteriana, que actualmente es una preocupación mundial a nivel de salud pública.

Se evidencia un aumento del 10\% en el porcentaje de usuarios que consultan al personal de las droguerías similar a lo reportado por Jaramillo et al. (5), CONPES (14) y Unión Temporal Econometría (28). Este aumento se da en todos los estratos socioeconómicos, sin importar le nivel educativo. Esto es favorable porque se reconoce la droguería como el sitio indicado donde están las personas conocedoras del uso adecuado de medicamentos; lo peligroso de esta buena práctica radica en que no todos los que trabajan en las droguerías tienen los estudios necesarios, tal como se afirma en el documento CONPES (14).

El comportamiento de los usuarios al comprar medicamentos también está influenciado por la publicidad, pues 3 de los 4 medicamentos que más adquieren los usuarios de Villavicencio tienen gran pauta publicitaria.

Los datos obtenidos en el estudio evidencian que el consumo de antibióticos en Villavicencio es 3.5 veces mayor con respecto a otras ciudades del Eje Cafetero en 2013 (14) y el estudio en cinco ciudades colombianas (5). Este elevado consumo de antibióticos preocupa por las consecuencias que trae su uso inadecuado, más aún cuando no son recomendados por el médico.

La adquisición de los medicamentos está muy influenciada porlasintomatología de las enfermedades, impulsando a los usuarios de las droguerías a consultar y comprar lo que les recomienda cualquier persona, sin importar el nivel educativo. Los usuarios de mayor estrato socioeconómico acostumbran a comprar medicamentos que ya saben que les sirven o los habían probado antes, comportamiento explicado, posiblemente, porque su entorno le facilita tener acceso a información de mejor calidad.

Los usuarios de las droguerías del estudio se caracterizan por darle poca importancia a la opinión del médico, comprando los medicamentos dependiendo de cómo consideran su salud; quienes más siguen las orientaciones de los médicos son los estratos socioeconómicos bajos. Los usuarios dan voto de confianza a las personas cercanas, pensando que la experiencia de los otros puede ser similar a la propia, sin tener en cuenta la existencia de sintomatología similar para enfermedades diferentes, 
lo que puede generar complicaciones. Además, al tratar únicamente la sintomatología, se enmascara Vol. 13 No. 2 la enfermedad de base, como puede ser el caso de la Jul - Dic 2016 hipertensión (29).

No consultar a un profesional de la salud (médico) representa, para el usuario de las droguerías, un ahorro, situación que se torna más crítica cuando se evidencia que el mayor porcentaje de usuarios de las droguerías de Villavicencio se ubica en el segundo estrato socioeconómico $(8,19,30)$, evidenciando la necesidad de sensibilizar a la comunidad en el uso adecuado de medicamentos.

\section{CONCLUSIONES}

Los hábitos de compra de medicamentos de los usuarios de droguerías se relacionan con la baja escolaridad, el nivel socioeconómico bajo y el fácil acceso a los medicamentos sin formula médica, siendo estos los factores contribuyentes para la automedicación.

La comercialización de los medicamentos se ve incrementada con la sensación de bienestar que producen, generando que las personas compren productos conocidos.

La compra de medicamentos generada por los altos índices de automedicación -evidenciada en el estudio-, puede ser un mecanismo de defensa frente a las sintomatologías de las enfermedades, facilitada por la forma de comercializar estos productos en Colombia.

Una de las razones que influye en la adquisición de los medicamentos es el fácil acceso a la información, lo que hace que el usuario este más pero no necesariamente mejor informado. En el estudio se evidencia que la mayoría de los usuarios que adquiere medicamentos consulta a otras personas (no necesariamente a los expertos) antes de tomarlos.

\section{CONFLICTO DE INTERESES}

El autor declara no tener ningún conflicto de intereses. 
ISSN 1794-9831

E-ISSN 2322-7028 Vol. 13 No. 2 Jul - Dic 2016 Cúcuta, Colombia

\section{REFERENCIAS BIBLIOGRÁFICAS}

1. Comisión Técnica Subregional para la Política de Acceso a Medicamentos / Organismo Andino de Salud Convenio Hipólito Unanue. Política Andina de Medicamentos. 1 a ed. Lima, Peru: Organismo Andino de Salud - Convenio Hipólito Unanue; 2009. [consultado 25 de marzo de 2016]; (24): 1-28. Disponible en: http://www. orasconhu.org/documentos/Politica\%20andina\%20de\%20medicamentos\%202009.pdf

2. Vélez CP. Rompiendo la tradición: De describir la conducta de compra a comprender al consumidor. Revista Pensamiento y gestión [Internet]. 2007 [consultado 16 de marzo de 2016]; (24): 1-28. Disponible en: http:// rcientificas.uninorte.edu.co/index.php/pensamiento/article/viewFile/3472/2235

3. Arboleda A, Alonso J. Determinantes de una elección errada al escoger medicamentos de venta libre. Revista Innovar Journal [Internet]. 2010 [consultado 15 de marzo de 2016]; 20(38): 175-189. Disponible en: http:// revistas.unal.edu.co/index.php/innovar/article/view/22397/34719

4. Uema S, Vega E, Briñón M. La problemática de la publicidad sobre los medicamentos y la medicalización. Córdoba. Centro de información sobre medicamentos. [Internet]. Córdoba, Argentina Universidad Nacional de Córdoba; 2008 [consultado 8 de diciembre de 2015]. Disponible en: http://www.cime.fcq.unc.edu.ar/boletin\%20 publicidad\%20VL\%202006-08.pdf

5. Jaramillo L, Orozco J, Sánchez R, Cárdenas M, García O. Estudio sobre uso y prescripción de medicamentos en cinco ciudades colombianas. [Internet]. Bogotá D.C.: Ministerio de la Proteccion Social; 2004 [consultado 10 de abril de 2016]. Disponible en: http://apps.who.int/medicinedocs/es/m/abstract/Js19006es/

6. Villegas F, Nasner K, Buitrago D, Cruz S, Ruden S, Bedolla J. Patrón de automedicación en la zona urbana de la ciudad de Pereira (Colombia) en el trimestre marzo- mayo 2013. Investigaciones Andina [Internet]. 2013[consultado 27 de marzo 2016];16(29): 1073-1085. Disponible en: http://www.scielo.org.co/pdf/inan/ v16n29/v16n29a07.pdf

7. García AJ. Caracterización epidemiológica del consumo de medicamentos por la población adulta de Cuba: 2007-2010. [Tesis doctoral]. Cuba: Escuela Nacional de Salud Pública; 2012.

8. De Pablo MM. La automedicación en la cultura universitaria. Rev. Invest [Internet]. 2011 [consultado 15 de abril de 2016]; 35(73): 219-240. Disponible en: http://www.scielo.org.ve/scielo.php?script=sci_arttext\&pid $=$ S1010-29142011000200011

9. The Nielsen Company. Nueva era, nuevo consumidor. Como y porque compramos al rededor del mundo. [Internet]. 2013 [consultado 24 de junio de 2016]; p. 17-19. Disponible en: http://www.nielsen.com/content/ dam/corporate/mx/reports/2013/NUEVA\%20ERA\%20NUEVO\%20CONSUMIDOR-REPORTE\%202013.pdf

10. Díaz N, Payán MA, Pérez AM. Aproximación psicológica al comportamiento de automedicación. Rev. Costarric Psicol [Internet]. 2014 [consultado 10 de abril de 2016]; 33(1): 17-29. Disponible en: http://dialnet.unirioja.es/ servlet/articulo? codigo $=4836496$

11. Martínez G, Martínez L, Rodríguez M. Características del consumo de medicamentos de venta libre en una población de adultos de la ciudad de Medellín (Colombia). Rev. Salud Uninorte Barranquilla [Internet]. 2013 [consultado 10 de abril de 2016]; 29(3): 360-367. Disponible en: http://www.scielo.org.co/pdf/sun/v29n3/ v29n3a02.pdf

12. Baes C. Uso racional del medicamento. Fundamento de la intervención administrativa en el sector farmacéutico. [Tesis doctoral en internet]. Granada: Universidad de Granada; 2010 [consultado 6 de abril de 2016]. Disponible en: http://hera.ugr.es/tesisugr/19557243.pdf

13. Arboleda A, Alonso J. Determinantes de una elección errada al escoger medicamentos de venta libre. Revista Innovar Journal [Internet]. 2010 [consultado 15 de marzo de 2016]; 20(38): 175-189. Disponible en: http:// revistas.unal.edu.co/index.php/innovar/article/view/22397/34719

14. Republica de Colombia, Departamento de Planeación Nacional, Consejo Nacional de Política Económica y Social (CONPES). Documento Conpes Social 155. Politica Farmaceutica Nacional. Version aprobada. [Internet]. 2012 [consultado 5 de abril de 2016]; 49 p. Disponible en: https://www.minsalud.gov.co/Documentos\%20y\%20 Publicaciones/Politica\%20Farmac\%C3\%A9utica\%20Nacional.pdf

15. Arnau J, Vallano A. Estudio Utilización de Medicamentos. Revi. Medicamentos y Salud. Lima. [Internet]. 2013 [consultado 1 de abril de 2016]; 78-82. Disponible en: http://evirtual.uaslp.mx/FCQ/farmaciahospitalaria/ Documents/EUM.pdf

16. Reyes E. Estudio de uso de medicamentos: representaciones sociales asociadas al medicamento y su consumo, en mujeres gestantes en el Hospital del Sur, Empresa Social del Estado de Bogotá. [Tesis maestría]. Bogotá D.C.: Universidad Nacional de Colombia; 2011.

17. Martín A, Sardón M, Iglesias H, Sáenz M, Miron J. Información sobre medicamentos y automedicación en las 
redes sociales. Rev. cuba. inf. cienc. salud [Internet] 2014 [consultado 28 de marzo de 2016]; 25(2): 145-156. Disponible en: http://scielo.sld.cu/scielo.php?script=sci_arttext\&pid=S2307-21132014000200002

18. García-Milian AJ, Alfonso-Carbonell L, López-Puig P, León-Cabrera P. Definición de consumo de medicamentos y su patrón. Horizonte Sanitario. [Internet].2015 [consultado 10 de abril de 2016]; 12(3): 79-83. Disponible en: http://revistas.ujat.mx/index.php/horizonte/article/view/71/39

19. Departamento Administrativo Nacional de Estadística (DANE). Demografía y Población. Proyecciones de Población; [Internet]. 2016 [consultado 22 de junio de 2016]. Disponible en: http://www.dane.gov.co/index. $\mathrm{php} /$ poblacion-y-demografia/proyecciones-de-poblacion

20. República de Colombia, Ministerio de Salud. Resolución No. 008430 de 1993, octubre 4, por la cual se establecen las normas científicas, técnicas y administrativas para la investigación en salud. Bogotá D.C.: Ministerio de Salud; 1993.

21. Espinoza K, Jofre V. Sobrecarga, apoyo social y autocuidado en cuidadores informales. Cienc. enferm. [Internet]. 2012 [consultado 3 de junio de 2016]; XVIII (2): 23-30. Disponible en: http://dx.doi.org/10.4067/ S0717-95532012000200003

22. Salmerón-Rubio S, García-Delgado P, Iglésias-Ferreira P, Mateus-Santos H, Martínez-Martínez F. Medida del conocimiento del paciente sobre su medicamento en farmacia comunitaria en Portugal. Ciência \& Saúde Coletiva [Internet]. 2015 [consultado 20 de marzo de 2016]; 1(20). Disponible en: http://www.scielo.br/pdf/csc/ v20n1/es_1413-8123-csc-20-01-00219.pdf

23. Hernández M, Delgado E, Munuera J. Elección del lugar de compra de medicamentos y parafarmacia. Distribución y Consumo. [Internet]. sep-oct 2001 [consultado 8 de abril de 2016]; p. 92-99. Disponible en: http://www.mercasa.es/files/multimedios/1309262780_DYC_2001_59_92_99.pdf

24. Alucema A, Chavarria N, Valdes M. Patrones de automedicación en clientes de una farmacia comunitaria de la ciudad de Antofagasta. Rev Journal of Pharmacy \& Pharmacognosy Research [Internet]. 2013[consultado 29 de marzo de 2016]; 1(2):54-63. Disponible en: http://jppres.com/jppres/patrones-de-automedicacion-enantofagasta/

25. Puig R, Perramon C, Yahni C, Garcia A. Establecimiento de los conocimientos, actitudes y opiniones de la población sobre el uso racional de medicamentos. Aten Primaria. [Internet]. 2015 [consultado 20 de marzo de 2016];47 (7): 446-455. Disponible en: http://ac.els-cdn.com/S0212656714003886/1-s2.0-S0212656714003886main.pdf?_tid=1756cc50-340e-11e6-90bf-00000aacb35f\&acdnat=1466114760_154f538706aa2c83b20285a59 $97 \mathrm{e} 2639$

26. Waldo J, Solis G, Campalans E, Moya Y. Automedicación en clubes de adulto mayor de la ciudad de valparaíso. Rev. Chil Salud Publica [Internet]. 2014 [consultado 2 de marzo de 2016]; 18(3): 274-285. Disponible en: http:// www.revistasaludpublica.uchile.cl/index.php/RCSP/article/viewFile/33977/35700

27. Organización Panamericana de Salud (OPS), Organización Mundial de la Salud (OMS). Propuesta regional para implementación de una estrategia nacional de uso racional de medicamentos. Documento actualizado en edición final. OPS/OMS; 2011.

28. República de Colombia, Ministerio de la Protección Social. Programa de reorganización, diseño y modernización de las redes de prestación de servicios de salud. Colombia: Unión Temporal Econometría; 2011.

29. Capítulo 23. Enfermedades crónicas con implicaciones nutricionales. En: Latham M. Nutrición humana en el mundo en desarrollo. Colección FAO: Alimentación y Nutrición. № 29. [Internet]. Roma: Organización de las Naciones Unidas para la Agricultura y la Alimentación (FAO); 2002 [consultado 7 de marzo de 2016]; p. 219236. Disponible en: http://www.fao.org/docrep/006/w0073s/w0073s0r.htm

30. Ramos J. Caracterización de la práctica de automedicación en la población residente del distrito de Ate de la provincia de Lima - marzo 2014. [Tesis doctoral]. Lima (P): Universidad Nacional Mayor de San Marcos; 2014. 